\title{
Anaplasmose : un diagnostic à évoquer après morsure de tique
}

\section{Anaplasmosis: A Diagnosis to Evoke after a Tick Bite}

\author{
C.E. Lavoignet $\cdot$ P. Le Borgne $\cdot$ H. Slimani
}

Reçu le 26 juillet 2017; accepté le 26 octobre 2017

(C) SFMU et Lavoisier SAS 2017

\section{Introduction}

Le nord-est de la France, en particulier l'Alsace, est une région endémique pour des pathologies infectieuses transmises par morsure de tiques du genre Ixodes Ricinus telles que la maladie de Lyme ou l'encéphalite à tiques. D'autres pathologies infectieuses doivent être évoquées en cas d'exposition aux tiques. Nous rapportons ici le cas d'une patiente chez laquelle a été diagnostiqué une anaplasmose granulocytique humaine, transmise après morsure d'une tique.

\section{Observation}

Une patiente de 53 ans, originaire du sud de l'Alsace, sans antécédent, s'est présentée aux urgences pour asthénie intense, perte d'appétit et syndrome fébrile évoluant depuis près de dix jours. Elle rapportait une morsure de tique, à la jambe, 18 jours avant la consultation aux urgences, et survenue après une randonnée dans le massif des Vosges. La patiente n'avait présenté aucun signe cutané au décours. Le médecin traitant, consulté au préalable avait évoqué une possible borréliose de Lyme bien que le tableau soit peu caractéristique en l'absence d'érythème migrant caractéristique. Une antibiothérapie par amoxicilline a été prescrite. Malgré ce traitement, la patiente restait fébrile, avec une asthénie marquée, des myalgies et des arthralgies diffuses. La patiente présentait par ailleurs une céphalée intense, intermittente, associée à des nausées et des vomissements, sans autre signe digestif ou urinaire. L'examen aux urgences

\footnotetext{
C.E. Lavoignet $(\square) \cdot$ H. Slimani

Service des urgences, hôpital Nord-Franche-Comté, 100 route de Moval, CS 10499 Trévenans, 90015 Belfort, France e-mail : charles-eric.lavoignet@hnfc.fr

P. Le Borgne

Service des urgences, hôpitaux universitaires de Strasbourg,

1 avenue Molière, 67200 Strasbourg
}

ne retrouvait pas de lésion cutanée ou de trace visible de morsure. La patiente ne rapportait aucun voyage récent. L'examen clinique ne retrouvait pas de signe d'infection des voies respiratoires. Les paramètres vitaux mesurés à l'admission aux urgences montraient une pression artérielle à $110 / 85 \mathrm{mmHg}$, une fréquence cardiaque à $85 / \mathrm{min}$, une saturation en oxygène à $98 \%$ et une température à $39,3^{\circ} \mathrm{C}$. Le bilan biologique initial montrait un taux de leucocytes à $1,86 \mathrm{G} / 1$ avec des polynucléaires neutrophiles à $1,1 \mathrm{G} / 1$, une thrombopénie $96 \mathrm{G} / \mathrm{l}$, une éosinopénie à 0 élément $/ \mathrm{mm}^{3}$, un taux de $C$ réactive protéine à $76 \mathrm{mg} / \mathrm{l}$, une cytolyse hépatique avec TGO à $312 \mathrm{U} / 1(\mathrm{~N}<35)$ et TGP $422 \mathrm{U} / 1(\mathrm{~N}<59)$. La bilirubine et les phosphatases alcalines étaient normales. La fonction rénale n'était pas altérée. Une radiographie pulmonaire ne retrouvait pas de foyer de condensation alvéolaire et la bandelette urinaire était négative. Sur la base de ces éléments anamnestiques et cliniques associant syndrome pseudogrippal, leuconeutropénie, thrombopénie et cytolyse hépatique après exposition à une morsure de tique récente, le médecin urgentiste a évoqué le diagnostic d'anaplasmose granulocytique humaine. Un traitement par doxycycline à la dose de $200 \mathrm{mg}$ par jour a été débuté par voie intraveineuse en raison de l'existence de vomissements. La fièvre a régressé en 24 heures et les céphalées ainsi que les douleurs diffuses ont disparues dans les trois jours suivant l'introduction de l'antibiothérapie. La patiente a pu rentrer à domicile à $\mathrm{J} 2$ avec un relais par doxycycline per os pour une durée totale de dix jours. Une sérologie pour Anaplasma phagocytophylum prélevée à $\mathrm{J} 1$ est revenue négative pour les IgM et les IgG. Une PCR Anaplasma phagocytophilum prélevée à J3 d'antibiothérapie, était revenue négative. En revanche, la patiente a présenté une séroconversion avec l'apparition d'IgG à trois mois de l'épisode confirmant, a posteriori, le diagnostic d'anaplasmose granulocytique humaine.

\section{Discussion}

Les tiques du genre Ixodes ricinus sont largement répandues en Europe, excepté sur le pourtour méditerranéen, et 
sont responsables de la transmission de nombreuses pathologies infectieuses. Les plus fréquentes dans le nord-est de la France sont la borréliose de Lyme, l'encéphalite à tique et l'anaplasmose [1-3]. En Europe, le premier cas d'anaplasmose humaine a été décrit en Slovénie en 1997 [1] alors que le premier cas français a été décrit en 2003 [2]. La pathologie est endémique en Europe Centrale et dans les pays nordiques [2]. Elle semble s'étendre progressivement vers l'Europe de l'Ouest en atteignant notamment l'Est de la France où elle est endémique en Alsace et dans le massif des Vosges [4,5], régions dans lesquelles la prévalence de la maladie de Lyme est également élevée [6]. La bactérie responsable de l'anaplosmose granulocytique humaine est Anaplasma phagocytophylum. Les tiques du genre Ixodes ricinus constituent le principal vecteur connu à la fois pour la maladie de Lyme et l'anaplasmose, ce qui explique la répartition géographique similaire de ces deux pathologies [6]. Le réservoir naturel de l'anaplasmose semble être représenté par les mammifères parmi lesquels l'Homme peut être un hôte accidentel à l'occasion d'une morsure de tique. La maladie survient principalement entre avril et octobre au sein d'une population exposée : activités forestières de loisir ou professionnelles. La durée d'incubation varie entre 7 et 21 jours. L'anaplasmose se manifeste chez l'Homme par des signes non spécifiques avec un syndrome pseudogrippal associant fièvre, arthromyalgies diffuses, céphalées, asthénie intense et des signes digestifs non spécifiques. Contrairement à la borréliose de Lyme, il n'existe pas d'érythème migrant, centrifuge et caractéristique, autour du point d'inoculation. La biologie montre classiquement une leuconeutropénie, une thrombopénie, une cytolyse hépatique [7]. Des agglomérats bactériens ou morulae peuvent être inconstamment observés sur le frottis sanguin au sein du cytoplasme des polynucléaires neutrophiles et sont pathognomoniques [7]. Le diagnostic peut être confirmé par sérologie, mais la séroconversion est tardive et survient généralement au-delà de la $4^{\mathrm{e}}$ semaine. Enfin, la PCR est l'examen le plus sensible et le plus spécifique [7]. Un prélèvement réalisé à distance du début de l'antibiothérapie peut toutefois expliquer, dans notre cas, le résultat négatif. L'évolution clinique peut être spontanément favorable, ce qui rend le recensement des cas relativement difficile. Près d'une dizaine de cas seulement sont diagnostiqués en Alsace chaque année, chiffre qui tend toutefois à se majorer sur les deux dernières décennies [4]. Néanmoins, la prévalence de la maladie est probablement sous-estimée à cause du caractère non spécifique des symptômes et en raison de l'évolution fréquemment favorable sans traitement spécifique. La mortalité peut en revanche atteindre $10 \%$ parmi les cas hospitalisés [7]. Elle fait suite à des complications graves telles que le choc septique, la coagulation intravasculaire disséminée ou une défaillance multiviscérale qui peuvent repré- senter plus de $36 \%$ des cas hospitalisés [8]. Le traitement de l'anaplasmose repose sur une antibiothérapie par doxycycline à la dose de $200 \mathrm{mg}$ par jour pour une durée de 7 à 14 jours. L'encéphalite virale à tique constitue un autre diagnostic différentiel à évoquer. Cette pathologie due au virus TBE (tick-borne encephalitis) est également transmise par les tiques du genre Ixodes et plusieurs foyers endémiques sont décrits en Alsace. La symptomatologie est proche des signes observés dans l'anaplasmose avec l'existence d'un syndrome pseudogrippal, suivi quelques jours plus tard d'un tableau neurologique variable (céphalées intenses, méningite, encéphalite, méningoradiculite). La prévalence d'Anaplasma phagocytophylum chez Ixodes ricinus en France est en moyenne de 1,25 à $5 \%[9,10]$. Ce chiffre est variable selon les zones géographiques et pourrait atteindre 2 à $45 \%$ dans certaines zones endémiques [9]. La séroprévalence de Anaplasma phagocytophylum est estimée à près de $5 \%$ dans les populations exposées aux morsures de tiques [11]. À titre de comparaison, la séroprévalence pour la maladie de Lyme peut atteindre $26,9 \%$ dans le nord-est de la France contre $14 \%$ en moyenne pour l'ensemble de la France [11]. La séroprévalence pour l'encéphalite à tique serait estimée à $3,4 \%$ dans la même population [11].

En conclusion, une morsure de tique doit toujours être recherchée en cas de syndrome fébrile inexpliqué. Les zoonoses transmises par les tiques sont fréquentes et parmi elles, la borréliose de Lyme est de loin la plus fréquente dans le nord-est de la France. Il est toutefois important d'évoquer le diagnostic d'anaplasmose, après morsure de tique, en présence d'un syndrome pseudogrippal et d'un tableau biologique caractéristique associant cytolyse hépatique, leuconeutropénie et thrombopénie, en particulier en cas de forme grave.

Liens d'intérêts : les auteurs ne déclarent aucun lien d'intérêt.

\section{Références}

1. Petrovec M, Lotric-Furlan S, Avsic-Zupanc T, et al (1997) Human diseases in Europe caused by a granulocytic Ehrlichia species. J Clin Microbiol 35:1556-9

2. Brouqui P, Bacellar F, Baranton G, et al (2004) ESCMID Study Group on Coxiella, Anaplasma, Rickettsia and Bartonella; European Network for Surveillance of Tick-Borne Diseases. Guidelines for the diagnosis of tick-borne bacterial diseases in Europe. Clin Microbiol Infect 10:1108-32

3. Jaussaud R, Magy N, Strady A, et al (2001). L'encéphalite virale à tiques. Rev Med Int 22:542-8

4. Edouard S, Koebel C, Goehringer F, et al (2012) Emergence of human granulocytic anaplasmosis in France. Ticks Tick Borne Dis 3:403-5 
5. Ismail N, McBride JW (2017) Tick-borne emerging infections: ehrlichiosis and anaplasmosis. Clin Lab Med 37:317-40

6. Mitschler A, Grange F, Lipsker D, et al (2004) Knowledge and prevention of tick-bite borreliosis: survey of the population in Alsace, an endemic area. Ann Dermatol Venereol 131:547-53

7. Koebel C, Kern A, Edouard S, et al (2012) Human granulocytic anaplasmosis in eastern France: clinical presentation and laboratory diagnosis. Diagn Microbiol Infect Dis 72:214-8

8. Dahlgren FS, Heitman KN, Drexler NA, et al (2015) Human granulocytic anaplasmosis in the United States from 2008 to
2012: a summary of national surveillance data. Am J Trop Med Hyg 93: 66-72

9. Blanco JR, Oteo JA (2002) Human granulocytic ehrlichiosis in Europe. Clin Microbiol Infect 8:763-72

10. Aubry C, Socolovschi C, Raoult D, et al (2016). Bacterial agents in 248 ticks removed from people from 2002 to 2013 . Ticks Tick Borne Dis 7:475-81

11. Thorin C, Rigaud E, Capek I, et al (2008) Seroprevalence of Lyme Borreliosis and tick-borne encephalitis in workers at risk, in eastern France. Med Mal Infect 38:533-42 